\title{
Dynamics of Dipoles and Quantum Phases in Noncommutative Coordinates
}

\author{
Ömer F. Dayl \\ Physics Department, Faculty of Science and Letters, Istanbul Technical University, TR-34469 Maslak-Istanbul, \\ Turkey, \\ and \\ Feza Gürsey Institute, P.O. Box 6, TR-34684, Çengelköy-Istanbul, Turkey.
}

\begin{abstract}
The dynamics of a spin-1/2 neutral particle possessing electric and magnetic dipole moments interacting with external electric and magnetic fields in noncommutative coordinates is obtained. Noncommutativity of space is interposed in terms of a semiclassical constrained Hamiltonian system. The relation between the quantum phase acquired by a particle interacting with an electromagnetic field and the (semi)classical force acting on the system is examined and generalized to establish a formulation of the quantum phases in noncommutative coordinates. The general formalism is applied to physical systems yielding the Aharonov-Bohm, Aharonov-Casher, He-McKellar-Wilkens and Anandan phases in noncommutative coordinates. Bounds for the noncommutativity parameter $\theta$ are derived comparing the deformed phases with the experimental data on the Aharonov-Bohm and Aharonov-Casher phases.
\end{abstract}

\section{Introduction}

The wave function of a particle encircling the sources of electromagnetic fields may acquire a phase factor which cannot be removed by gauge or phase transformations. The first predicted quantum phase is the well-established Aharonov-Bohm (AB) phase 1. Later for spin-1/2 neutral particles possessing magnetic dipole moment a similar effect was predicted by Aharonov-Casher (AC) 2. Although, it has not been experimentally verified yet HeMcKellar and Wilkens (HMW) derived the dual of the AC phase for neutral particles with an electric dipole moment 3. In [4 Anandan studied the dynamics of a spin $-1 / 2$ neutral particle possessing both electric and magnetic dipole moments interacting with external electric and magnetic fields which leads to a quantum phase for a specific configuration $[5$. These quantum phases have already been studied in noncommutative space. For the $\mathrm{AB}$ phase in noncommutative coordinates there appeared different results depending on the velocity [6, , 7 . and independent of the velocity [8. However, for the AC [9, 10, HMW [1] and the Anandan [12] phases in noncommutative space similar results which explicitly depend on eigenvalues of the momentum operator $\hat{p}_{\mu}$, are obtained. In all these studies noncommutativity of the coordinates $r_{\mu}$ is mainly imposed by shifting the coordinates as $r_{\mu} \rightarrow r_{\mu}-\frac{i}{2 \hbar} \theta_{\mu \nu} \hat{p}^{\nu}$, where $\theta$ is the constant noncommutativity parameter. Within this approach one attains a $\theta$ deformed Schroedinger equation or equivalently a $\theta$ deformed Hamiltonian which are then employed to calculate the related quantum phases. Obviously, this scheme is very sensitive how the external fields depend on the coordinates $r_{\mu}$.

We are concerned with deriving the dynamical equations of a spin- $1 / 2$ particle possessing dipole moments interacting with electromagnetic fields in noncommutative coordinates as well as obtaining the $\theta$ deformed quantum phases in the framework of the semiclassical constrained Hamiltonian formalism proposed in [13. This semiclassical approach which was invented to study the Berry gauge fields 14 arising in spin dynamics, is suitable to study gauge systems in terms of field strengths without referring to the explicit realization of the gauge fields. Moreover, it is adequate to study spin-dependent dynamical systems in noncommutative coordinates as was shown in [15]. We briefly review the formalism of $[13$ in the next section. In the third section we first present the general formulation of deriving the equations of motion and semiclassical force in noncommutative coordinates and then apply it to a

\footnotetext{
${ }^{1}$ E-mail addresses: dayi@gursey.gov.tr and dayi@itu.edu.tr.
} 
spin-1/2 neutral particle possessing electric and magnetic dipole moments interacting with external electric and magnetic fields in noncommutative coordinates.

Our semiclassical constrained Hamiltonian formalism does not give rise to an effective Hamiltonian which can be utilized to discern the quantum phases in noncommutative coordinates. Instead, we are equipped with the equations of motion and the force acting on the system. Fortunately, in [16] it was shown that the quantum phase acquired by a particle interacting with an electromagnetic field is related to the classical force acting on the system. By generalizing this relation to noncommutative systems we will present a definition of the quantum phase in noncommutative space, which is velocity independent. We apply the formulation to obtain the $\mathrm{AB}$ phase and the Anandan phase in noncommutative coordinates. Obviously, from the Anandan phase the AC and HMW phases follow, respectively, for vanishing electric and magnetic dipole moments. We then argue that our formulation can be generalized to formulate the quantum phase attained by a particle interacting with the external gauge field taking values in the noncommutative $U(N)$. In the last section we compare the $\theta$ deformed $\mathrm{AB}$ and $\mathrm{AC}$ phases which we obtained with the experimental data to derive bounds on the noncommutativity parameter $\theta$.

\section{The semiclassical approach}

We would like to present briefly the semiclassical scheme established in 13 for studying spin dependent dynamical systems. We consider matrix observables which are functions of the classical phase space variables $\left(\pi_{\mu}, x_{\mu}\right)$, though they are allowed to be $\hbar$ dependent. One of the main ingredients is the semiclassical bracket of the observables $K(\pi, x)$ and $N(\pi, x)$ defined as

$$
\{K(\pi, x), N(\pi, x)\}_{C} \equiv \frac{-i}{\hbar}[K, N]+\frac{1}{2}\{K(\pi, x), N(\pi, x)\}-\frac{1}{2}\{N(\pi, x), K(\pi, x)\},
$$

which is obtained from the Moyal bracket by retaining the lowest two terms in $\hbar$. The first term is the ordinary commutator of matrices, it is not the quantum commutator. The others are the Poisson brackets defined as

$$
\{K(\pi, x), N(\pi, x)\} \equiv \frac{\partial K}{\partial x^{\nu}} \frac{\partial N}{\partial \pi_{\nu}}-\frac{\partial K}{\partial \pi_{\nu}} \frac{\partial N}{\partial x^{\nu}} .
$$

$K$ and $N$ may depend on $\hbar$, but only the two lowest order terms in $\hbar$ are detained in (1), so that it is a semiclassical approximation. The semiclassical bracket (11) and the semiclassical Hamiltonian $H(\pi, x)$ are employed to define

$$
\dot{K}(\pi, x)=\{K(\pi, x), H(\pi, x)\}_{C},
$$

where the dot over the observables indicates the time derivative. This designates the semiclassical dynamics.

\subsection{A semiclassical constrained Hamiltonian system}

To generate the semiclassical dynamics one substitutes the Poisson brackets with the semiclassical brackets (1) in the ordinary Hamiltonian dynamical relations. To achieve a systematic method of describing the dynamics in noncommutative space, we introduce the first-order matrix Lagrangian

$$
\mathcal{L}=\dot{r}^{\alpha}\left[\operatorname{Ip}_{\alpha} / 2+\rho \mathcal{A}_{\alpha}(r, p)\right]-\dot{p}^{\alpha}\left[\operatorname{Ir}_{\alpha} / 2-\xi \mathcal{B}_{\alpha}(r, p)\right]-H_{0}(r, p),
$$

in terms of the gauge fields $\mathcal{A}_{\alpha}, \mathcal{B}_{\alpha}$ which are in general matrix valued. $\rho, \xi$ are the coupling constants and $I$ denotes the unit matrix. In this work we only deal with three dimensional systems, so that $\alpha, \beta=1,2,3$. This Lagrangian leads to some constraints which are second class. To effectively set them to zero we employ the Dirac procedure by substituting the semiclassical bracket of observables (1) with the semiclassical Dirac bracket defined adequately. In fact one can calculate that the phase space variables satisfy the following semiclassical Dirac brackets

$$
\begin{aligned}
& \left\{r^{\alpha}, r^{\beta}\right\}_{C D}=\xi G^{\alpha \beta}-\rho \xi^{2}(G F G)^{\alpha \beta}+\cdots \\
& \left\{p^{\alpha}, p^{\beta}\right\}_{C D}=\rho F^{\alpha \beta}-\rho^{2} \xi(F G F)^{\alpha \beta}+\cdots \\
& \left\{r^{\alpha}, p^{\beta}\right\}_{C D}=\delta^{\alpha \beta}-\rho \xi(G F)^{\alpha \beta}+\cdots
\end{aligned}
$$


Here $(G F)^{\alpha \beta} \equiv G^{\alpha \gamma} F_{\gamma}^{\beta},(G F G)^{\alpha \beta} \equiv G^{\alpha \gamma} F_{\gamma \sigma} G^{\sigma \beta}$ and we defined the field strengths as

$$
\begin{aligned}
F_{\alpha \beta} & =\frac{\partial \mathcal{A}_{\beta}}{\partial r^{\alpha}}-\frac{\partial \mathcal{A}_{\alpha}}{\partial r^{\beta}}-\frac{i \rho}{\hbar}\left[\mathcal{A}_{\alpha}, \mathcal{A}_{\beta}\right] \\
G_{\alpha \beta} & =\frac{\partial \mathcal{B}_{\beta}}{\partial p^{\alpha}}-\frac{\partial \mathcal{B}_{\alpha}}{\partial p^{\beta}}-\frac{i \xi}{\hbar}\left[\mathcal{B}_{\alpha}, \mathcal{B}_{\beta}\right]
\end{aligned}
$$

Moreover, we deal with the gauge fields satisfying the conditions: $\mathcal{B}_{\beta}=\mathcal{B}_{\beta}(p) ; \mathcal{A}_{\alpha}=\mathcal{A}_{\alpha}(r) ;\left[\mathcal{A}_{\alpha}, \mathcal{B}_{\beta}\right]=0$. Observe that $r_{\alpha}$ and $p_{\alpha}$ should be considered as coordinates and the corresponding momenta, respectively. One can also show that the equations of motion of the phase space variables are

$$
\begin{array}{r}
\dot{r}^{\alpha}=\xi\left(\frac{\partial H_{0}}{\partial r^{\beta}}-\frac{i \rho}{\hbar}\left[\mathcal{A}_{\beta}, H_{0}\right]\right)\left(G^{\alpha \beta}-\rho \xi(G F G)^{\alpha \beta}+\cdots\right) \\
+\left(\frac{\partial H_{0}}{\partial p^{\beta}}-\frac{i \xi}{\hbar}\left[\mathcal{B}_{\beta}, H_{0}\right]\right)\left(\delta^{\alpha \beta}-\rho \xi(G F)^{\alpha \beta}+\cdots\right), \\
\dot{p}^{\alpha}=\left(\frac{\partial H_{0}}{\partial r^{\beta}}-\frac{i \rho}{\hbar}\left[\mathcal{A}_{\beta}, H_{0}\right]\right)\left(-\delta^{\alpha \beta}+\rho \xi(F G)^{\alpha \beta}+\cdots\right) \\
+\rho\left(\frac{\partial H_{0}}{\partial p^{\beta}}-\frac{i \xi}{\hbar}\left[\mathcal{B}_{\beta}, H_{0}\right]\right)\left(F^{\alpha \beta}-\rho \xi(F G F)^{\alpha \beta}+\cdots\right) .
\end{array}
$$

The method which we presented is valid for non-Abelian gauge fields which may be internal or external. To clarify this point let the gauge field $\mathcal{A}$ be Lie algebra valued, defined as $\mathcal{A}_{\alpha}=\mathcal{A}_{\alpha}^{n} T_{n}$. Here $T_{n}$ are the generators of a Lie algebra with the structure constants $f_{n m k}$ :

$$
\left[T_{n}, T_{m}\right]=f_{n m k} T_{k} .
$$

Hence, the field strength components are given as

$$
F_{\alpha \beta}^{n}=\frac{\partial \mathcal{A}_{\beta}^{n}}{\partial r^{\alpha}}-\frac{\partial \mathcal{A}_{\alpha}^{n}}{\partial r^{\beta}}-\frac{i \rho}{\hbar} f^{n m k} \mathcal{A}_{\alpha}^{m} \mathcal{A}_{\beta}^{k}
$$

The difference between internal and external gauge fields resides in the $\hbar$ dependence of the coupling constant $\rho[17$ : for an internal gauge field the coupling constant $\rho$ can be chosen to be linear in $\hbar$, but for an external gauge field the coupling constant $\rho$ does not possess any $\hbar$ dependence. In the latter case the field strength (11) is singular in the $\hbar \rightarrow 0$ limit.

\section{Dynamics in noncommutative space}

Inspecting the basic relations of the phase space variables (3)-(5) one can observe that to introduce noncommutativity of the coordinates $r_{\alpha}$ one can set $\xi=1$ and choose the gauge field $\mathcal{B}_{\alpha}$ appropriately so that its field strength yields

$$
G_{\alpha \beta}=\theta_{\alpha \beta}
$$

The noncommutativity parameter $\theta_{\alpha \beta}$ is constant and antisymmetric. Let us deal with the systems where the canonical Hamiltonian satisfies

$$
\frac{\partial H_{0}}{\partial p_{\alpha}}=\frac{p_{\alpha}}{m} ;\left[\mathcal{B}_{\alpha}, H_{0}\right]=0
$$

By keeping the terms which are first order in $\theta_{\alpha \beta}$ and second order in $\rho$ in (3)-(5), one can observe that the basic relations between the phase space variables are

$$
\begin{aligned}
& \left\{r^{\alpha}, r^{\beta}\right\}_{C D}=\theta^{\alpha \beta} \\
& \left\{p^{\alpha}, p^{\beta}\right\}_{C D}=\rho F^{\alpha \beta}-\rho^{2}(F \theta F)^{\alpha \beta}, \\
& \left\{r^{\alpha}, p^{\beta}\right\}_{C D}=\delta^{\alpha \beta}-\rho(\theta F)^{\alpha \beta} .
\end{aligned}
$$


The equations of motion of the phase space variables follow from (8), (9) as

$$
\begin{aligned}
\dot{r}^{\alpha} & =\left(\frac{\partial H_{0}}{\partial r^{\beta}}-\frac{i \rho}{\hbar}\left[\mathcal{A}_{\beta}, H_{0}\right]\right) \theta^{\alpha \beta}+\frac{p_{\beta}}{m}\left(\delta^{\alpha \beta}-\rho(\theta F)^{\alpha \beta}\right) \\
\dot{p}^{\alpha} & =\left(\frac{\partial H_{0}}{\partial r^{\beta}}-\frac{i \rho}{\hbar}\left[\mathcal{A}_{\beta}, H_{0}\right]\right)\left(-\delta^{\alpha \beta}+\rho(F \theta)^{\alpha \beta}\right)+\rho \frac{p_{\beta}}{m}\left(F^{\alpha \beta}-\rho(F \theta F)^{\alpha \beta}\right)
\end{aligned}
$$

by retaining up to the first and the second order terms, respectively, in $\theta$ and $\rho$. (17) can be utilized to write the momentum in terms of the velocity $\dot{r}_{\alpha}$ as

$$
p^{\alpha}=m\left(\delta^{\alpha \beta}+\rho(\theta F)^{\alpha \beta}\right) \dot{r}_{\beta}-\left(\frac{\partial H_{0}}{\partial r^{\beta}}-\frac{i \rho}{\hbar}\left[\mathcal{A}_{\beta}, H_{0}\right]\right) \theta^{\alpha \beta} .
$$

Making use of (19) one can derive the force acting on the system by keeping the terms at most linear in the velocity as

$$
\begin{aligned}
m \ddot{r}_{\alpha}= & -\left(\frac{\partial H_{0}}{\partial r^{\beta}}-\frac{i \rho}{\hbar}\left[\mathcal{A}_{\beta}, H_{0}\right]\right)\left(\delta_{\alpha \beta}-\rho(\theta F)_{\alpha \beta}\right)-\frac{i m}{\hbar} \theta_{\alpha \beta}\left[\frac{\partial H_{0}}{\partial r^{\beta}}-\frac{i \rho}{\hbar}\left[\mathcal{A}_{\beta}, H_{0}\right], H_{0}\right] \\
& +\frac{i m \rho}{\hbar} \theta_{\alpha \beta}\left(\left[F_{\beta \gamma}, H_{0}\right]+\left[\frac{\partial H_{0}}{\partial r^{\beta}}-\frac{i \rho}{\hbar}\left[\mathcal{A}_{\beta}, H_{0}\right], \mathcal{A}_{\gamma}\right]\right) \dot{r}_{\gamma} \\
& +m \theta_{\alpha \beta} \frac{\partial}{\partial r_{\gamma}}\left(\frac{\partial H_{0}}{\partial r^{\beta}}-\frac{i \rho}{\hbar}\left[\mathcal{A}_{\beta}, H_{0}\right]\right) \dot{r}_{\gamma}+\left(\rho F_{\alpha \beta}-\rho^{2}(\theta F F)_{\alpha \beta}\right) \dot{r}_{\beta} .
\end{aligned}
$$

In the commutative limit the force becomes

$$
\left.m \ddot{r}_{\alpha}\right|_{\theta=0}=-\frac{\partial H_{0}}{\partial r^{\alpha}}+\frac{i \rho}{\hbar}\left[\mathcal{A}_{\alpha}, H_{0}\right]+\rho F_{\alpha \beta} \dot{r}_{\beta} .
$$

This is in accord with [4] where the relativistic case was considered.

\subsection{The dynamics of dipoles in noncommutative coordinates}

Let us deal with a neutral elementary particle of spin-1/2 possessing the magnetic and electric dipole moments $\boldsymbol{\mu}=\mu \boldsymbol{\sigma}$ and $\boldsymbol{d}=d \boldsymbol{\sigma}$, which moves in the external electric and magnetic fields $\boldsymbol{E}$ and $\boldsymbol{B}$. Here $\boldsymbol{\sigma}$ are the Pauli matrices and $\mu=\hbar \gamma_{M}, d=\hbar \delta_{D}$ where $\gamma_{M}, \delta_{D}$ are constants. In [4] the dynamics of this system in ordinary (commutative) space was studied in the low energy limit. The interaction Hamiltonian employed in 4 can also be derived in terms of Foldy-Wouthuysen transformations from the underlying relativistic equation 12 , which can be adapted to our approach by choosing the canonical Hamiltonian as

$$
H_{0}=\frac{p^{2}}{2 m}-\boldsymbol{\mu} \cdot \boldsymbol{B}-\boldsymbol{d} \cdot \boldsymbol{E}
$$

and introducing the vector field

$$
\mathcal{A}=-\mu \times E+d \times B
$$

Obviously, time reversal symmetry is violated for a nonvanishing $\boldsymbol{d}$. Plugging (22) and (23) into (6) with the coupling constant $\rho=-1 / c$ leads to the related field strength:

$$
\begin{aligned}
F_{\alpha \beta}= & \epsilon_{\beta \sigma \gamma} \partial_{\alpha}\left(-\mu_{\sigma} E_{\gamma}+d_{\sigma} B_{\gamma}\right)-\epsilon_{\alpha \sigma \gamma} \partial_{\beta}\left(-\mu_{\sigma} E_{\gamma}+d_{\sigma} B_{\gamma}\right) \\
& -\frac{2}{\hbar c} \epsilon_{\alpha \beta \gamma}\left(-\mu E_{\gamma}+d B_{\gamma}\right)(\boldsymbol{\mu} \cdot \boldsymbol{E}+\boldsymbol{d} \cdot \boldsymbol{B})
\end{aligned}
$$

We can obtain the noncommutative version of this system in the framework outlined above. In fact, the full force acting on the particle in noncommutative space can be derived employing (22) and (24) in (20). The velocity dependent terms of the force (20) are related to the quantum phases which is the subject of the next section, so 
that here we only present the force for $\dot{\boldsymbol{r}}=0$ :

$$
\begin{aligned}
\left.f_{\alpha}(\theta)\right|_{\dot{\boldsymbol{r}}=0}= & \left(\frac{\partial}{\partial r_{\beta}}(\boldsymbol{\mu} \cdot \boldsymbol{B}+\boldsymbol{d} \cdot \boldsymbol{E})+\frac{2}{\hbar c} \epsilon_{\beta \sigma \gamma}(\boldsymbol{\mu} \times \boldsymbol{B}+\boldsymbol{d} \times \boldsymbol{E})_{\sigma}\left(-\mu E_{\gamma}+d B_{\gamma}\right)\right)\left(\delta_{\alpha \beta}+\frac{1}{c}(\theta F)_{\alpha \beta}\right) \\
& -\frac{2 m}{\hbar} \theta_{\alpha \beta}(\boldsymbol{\mu} \times \boldsymbol{B}+\boldsymbol{d} \times \boldsymbol{E}) \cdot \frac{\partial}{\partial r_{\beta}}(\mu \boldsymbol{B}+d \boldsymbol{E}) \\
& -\frac{4 m}{\hbar^{2} c} \theta_{\alpha \beta} \epsilon_{\beta \sigma \gamma}\left(\mu B_{\sigma}+d E_{\sigma}\right)\left(-\mu E_{\gamma}+d B_{\gamma}\right)(\boldsymbol{\mu} \cdot \boldsymbol{B}+\boldsymbol{d} \cdot \boldsymbol{E}) \\
& +\frac{4 m}{\hbar^{2} c} \theta_{\alpha \beta}(-\boldsymbol{\mu} \times \boldsymbol{E}+\boldsymbol{d} \times \boldsymbol{B})_{\beta}(\mu \boldsymbol{B}+d \boldsymbol{E}) \cdot(\mu \boldsymbol{B}+d \boldsymbol{E}) .
\end{aligned}
$$

For $\theta_{\alpha \beta}=0$ this coincides with the force given in [4].

In particular, considering $d=0$ and constant $\mu$ interacting with homogeneous external fields, the force (25) yields

$$
\left.\boldsymbol{f}(\theta)\right|_{\dot{\boldsymbol{r}}=0}=-\frac{2 \mu}{\hbar c}(\boldsymbol{\mu} \times \boldsymbol{B}) \times \boldsymbol{E}-\frac{4 m \mu^{2}}{\hbar^{2} c}\left[(\boldsymbol{\mu} \cdot \boldsymbol{B}) \boldsymbol{\theta} \times(\boldsymbol{B} \times \boldsymbol{E})+B^{2} \boldsymbol{\theta} \times(\boldsymbol{E} \times \boldsymbol{\mu})\right],
$$

where we introduced $\theta_{\alpha \beta}=\epsilon_{\alpha \beta \gamma} \theta_{\gamma}$ and neglected the terms of the order $1 / c^{2}$. One can observe that the force (26) is nonvanishing when both external electric and magnetic fields are present. In the commutative limit the force (26) may be detected [18. When the experimental data is available it may be used to set a bound on $\theta$ employing the scheme which will be discussed in the last section.

\section{The quantum phase from the (semi)classical force}

We would like to derive the quantum phases from the (semi)classical forces employing the relation given in [16]: The integral along a closed path $C$ of the classical force $f_{\alpha}$ acting on a particle of mass $m$ coupled to the Abelian gauge field $a_{\alpha}$ with the coupling constant $\eta$ leads to

$$
\frac{i}{\hbar} \oint_{C} f_{\alpha} d r_{\alpha}=-i \frac{d \Phi}{d t}
$$

where $\Phi$ is the quantum phase defined as

$$
\Phi=\frac{\eta}{\hbar} \oint_{C} a_{\alpha} d r_{\alpha}
$$

This may seem to be a controversy because generally on the path where the quantum phase is calculated there is no force acting on the particle. The force can be considered to be hypothetical as it was suggested in [16]. Nevertheless, it is an efficient procedure of obtaining the change in the action when a vector field is coupled to the system, even if the quantum treatment becomes complicated (see also [19]). It was also shown in [20] that the quantum phases and the equations of motion which lead to the force are interrelated.

When one deals with the non-Abelian gauge field $a_{\alpha} \equiv a_{\alpha}^{n} T_{n}$ where the generators of the Lie algebra $T_{n}$ satisfy the commutator (10), the related phase factor can be given by the path ordering $P$ which can be calculated as

$$
P \exp \left(\frac{-i \eta}{\hbar} \oint_{C_{\epsilon}} a_{\alpha} d r_{\alpha}\right)=1-\frac{i \eta}{2 \hbar} f_{\alpha \beta}\left(\boldsymbol{r}_{\epsilon}\right) s_{\epsilon}^{\alpha \beta}
$$

for an infinitesimal closed curve $C_{\epsilon}$ around the point $\boldsymbol{r}_{\epsilon}$. Here $s_{\epsilon}^{\alpha \beta}$ is the area of the infinitesimal surface spanned by $C_{\epsilon}$ and $f_{\alpha \beta}$ is the related non-Abelian field strength. Thus, for non-Abelian gauge fields we can generalize (27) as

$$
\frac{i}{\hbar} \oint_{C_{\epsilon}} f_{\alpha} d r_{\alpha}=-i \frac{d \Phi_{\epsilon}}{d t}
$$

where the infinitesimal phase is defined by

$$
\Phi_{\epsilon} \equiv \frac{\eta}{2 \hbar} f_{\alpha \beta}\left(\boldsymbol{r}_{\epsilon}\right) s_{\epsilon}^{\alpha \beta}
$$


Now, we would like to demonstrate that in the ordinary space our semiclassical approach is in accord with this formulation. Hence let us set $\mathcal{B}_{\alpha}=0$ and $H_{0}=p^{2} / 2 m$. Obviously, adding a potential term like $V(r)$ to the free Hamiltonian does not affect the quantum phase. The equations of motion (8), (9) give

$$
\dot{r}_{\alpha}=\frac{p_{\alpha}}{m}, \dot{p}_{\alpha}=\rho F_{\alpha \beta} \dot{r}^{\beta} .
$$

The integral of the force $m \ddot{r}_{\alpha}=\dot{p}_{\alpha}$ along the infinitesimal path $C_{\epsilon}$ around the point $\boldsymbol{r}_{\epsilon}$ yields

$$
\rho \oint_{C_{\epsilon}} F_{\alpha \beta} \dot{r}^{\beta} d r^{\alpha}=\rho F_{\alpha \beta}\left(\boldsymbol{r}_{\epsilon}\right) \oint_{C_{\epsilon}} \dot{r}^{\beta} d r^{\alpha}=-\frac{\rho}{2} F_{\alpha \beta}\left(\boldsymbol{r}_{\epsilon}\right) \frac{d s_{\epsilon}^{\alpha \beta}}{d t}=-\frac{\rho}{2} \frac{d}{d t}\left(F_{\alpha \beta}\left(\boldsymbol{r}_{\epsilon}\right) s_{\epsilon}^{\alpha \beta}\right),
$$

where we suppose that the infinitesimal loop is moving so that $d s_{\epsilon}^{\alpha \beta} / d t=\oint_{C_{\epsilon}}\left(\dot{r}_{\alpha} d r_{\beta}-\dot{r}_{\beta} d r_{\alpha}\right)$ is the rate of change of the area of the surface spanned by the curve $C_{\epsilon}$, and $F_{\alpha \beta}$ does not depend on time explicitly. Therefore, by substituting $\rho$ and $F_{\alpha \beta}$ with $\eta$ and $f_{\alpha \beta}$ the relation (29) follows. Except the points where $F_{\alpha \beta}$ is singular, one can extend the infinitesimal $\Phi_{\epsilon}$ to an integral over a finite surface $S$, whether the gauge fields $\mathcal{A}_{\alpha}$ is Abelian or non-Abelian. However, when the gauge field $\mathcal{A}$ is Abelian the infinitesimal phase can be written as

$$
\Phi_{\epsilon}=\frac{\rho}{\hbar}(\nabla \times \mathcal{A}) \cdot s_{\epsilon}
$$

where the infinitesimal vector area is defined by $\boldsymbol{s}_{\epsilon} \equiv \oint_{C_{\epsilon}} \boldsymbol{r} \times d \boldsymbol{r} / 2$. Extension of (33) to a finite surface $S$ leads to

$$
\Phi=\frac{\rho}{\hbar} \int_{S}(\nabla \times \mathcal{A}) \cdot d \boldsymbol{s}=\frac{\rho}{\hbar} \oint_{C} \mathcal{A} \cdot d \boldsymbol{r}
$$

which is the quantum phase.

\section{The quantum phases in noncommutative coordinates}

We would like to generalize the procedure outlined above to define the quantum phases in noncommutative coordinates. We adopt (29) as the definition of the quantum phase for a given force: we set $H_{0}=p^{2} / 2 m$ which is adequate for the configurations which we will consider, so that the force in noncommutative coordinates (20) becomes

$$
f_{\alpha}(\theta)=\left(\rho F_{\alpha \beta}-\rho^{2}(\theta F F)_{\alpha \beta}\right) \dot{r}^{\beta} .
$$

Hence, calculation of the integral of the $\theta$-deformed force (35) along the infinitesimal closed path $C_{\epsilon}$, using the approach employed in the ordinary case (32),

$$
\frac{i}{\hbar} \oint_{C_{\epsilon}} f_{\alpha}(\theta) d r_{\alpha}=-i \frac{d \Phi_{\epsilon}(\theta)}{d t},
$$

yields the definition of the $\theta$ deformed quantum phase as

$$
\Phi_{\epsilon}(\theta)=\frac{\rho}{2 \hbar}\left(F_{\alpha \beta}-\rho(\theta F F)_{\alpha \beta}\right) s_{\epsilon}^{\alpha \beta} .
$$

We can extend (37) to an integral over a finite surface $S$, as far as one does not encounter any singularity of the integrand:

$$
\Phi(\theta)=\frac{\rho}{2 \hbar} \int_{S} \epsilon^{\alpha \beta \gamma}\left(F_{\alpha \beta}-\rho(\theta F F)_{\alpha \beta}\right) d s_{\gamma} .
$$

When we deal with an Abelian gauge field (38) is the deformation of the quantum phase (34) at the first order in $\theta$. However, for a non-Abelian gauge field we should consider (37). One of the important properties of the original quantum phases is their independence of the velocity. Our formulation of $\theta$ deformed phases respects this property. 


\subsection{The AB phase}

Let an electron beam encircles a solenoid placed at the origin whose symmetry axis is along $r_{3}$. Thus, we set $H_{0}=p^{2} / 2 m, \rho=e / c$ and the field strength vanishes outside the solenoid but inside it is given with the constant magnetic field $B$ as $F_{i j}=\epsilon_{i j} B$. Here $i, j=1,2$. By letting the $r_{1} r_{2}$ coordinates be noncommuting, $\theta_{i j}=\epsilon_{i j} \theta$, one gets the $\theta$ deformed $\mathrm{AB}$ phase from (38) as

$$
\Phi_{A B}(\theta)=\frac{e B S}{\hbar c}\left(1+\frac{e B \theta}{c}\right)=\Phi_{A B}\left(1+\frac{e B \theta}{c}\right),
$$

where $S$ is the area enclosed in the solenoid and $\Phi_{A B}=\frac{e B S}{\hbar c}$ is the $\mathrm{AB}$ phase related to the flux $B S$ through the solenoid. This is in accord with the result of [8] which was obtained by generalizing the effective Hamiltonian obtained in a particular gauge.

\subsection{Dipoles interacting with electromagnetic fields}

We would like to obtain the $\theta$ deformed quantum phases for the dipoles interacting with external electric and magnetic fields whose dynamics was discussed before, (22)-(26). We deal with the configuration where $\boldsymbol{\mu} \cdot \boldsymbol{B}=$ $0 ; \boldsymbol{\mu} \cdot \boldsymbol{E}=0 ; \boldsymbol{d} \cdot \boldsymbol{B}=0 ; \boldsymbol{d} \cdot \boldsymbol{E}=0$. Under these conditions the Hamiltonian (22) yields $H_{0}=p^{2} / 2 m$ and the gauge field (23) satisfies: $\left[\mathcal{A}_{\alpha}, \mathcal{A}_{\beta}\right]=0$. We further choose $\boldsymbol{\mu}=\mu \hat{r}_{3} ; \boldsymbol{d}=d \hat{r}_{3}$ and let there be no change in the dipoles along the external fields: $\boldsymbol{E} \cdot \boldsymbol{\nabla} \mu=0 ; \boldsymbol{B} \cdot \boldsymbol{\nabla} d=0$. In this framework the field strength (24) can be written as

$$
F_{i j}=\epsilon_{i j}(-\mu \boldsymbol{\nabla} \cdot \boldsymbol{E}+d \boldsymbol{\nabla} \cdot \boldsymbol{B}) .
$$

Let the external electromagnetic fields be in the radial direction such that $\boldsymbol{\nabla} \cdot \boldsymbol{E}$ and $\boldsymbol{\nabla} \cdot \boldsymbol{B}$ vanish except in the small regions around the third axes:

$$
\nabla \cdot \boldsymbol{E}=\frac{\lambda_{e}}{s^{\prime}} ; \nabla \cdot \boldsymbol{B}=\frac{\lambda_{m}}{s^{\prime \prime}} .
$$

Here $s^{\prime}$ and $s^{\prime \prime}$ indicate, respectively, the areas of the regions where $\boldsymbol{\nabla} \cdot \boldsymbol{E}$ and $\boldsymbol{\nabla} \cdot \boldsymbol{B}$ do not vanish, though usually one uses the limit values

$$
\lim _{s^{\prime} \rightarrow 0} \frac{1}{s^{\prime}}=\lim _{s^{\prime \prime} \rightarrow 0} \frac{1}{s^{\prime \prime}}=\delta\left(r_{1}\right) \delta\left(r_{2}\right) .
$$

$\lambda_{e}$ is the charge per unit length of the straight line charge distribution along the third axis. Similarly, $\lambda_{m}$ is related to magnetic monopoles or dipoles. The $\theta=0$ part of the phase (38) yields

$$
\Phi_{A}=\frac{-1}{\hbar c} \int F_{12} d r_{1} d r_{2}=\frac{1}{\hbar c}\left(\mu \lambda_{e}-d \lambda_{m}\right),
$$

whether one uses (41) with the areas $s^{\prime}, s^{\prime \prime}$ or their limits (42). However, if one employs (42) instead of the areas $s^{\prime}, s^{\prime \prime}$ attributed to the line of electric and magnetic charges in (41), the $\theta$ dependent part of the phase (38) will not be well defined. Hence, we keep $s^{\prime}, s^{\prime \prime}$ and from (38) obtain the Anandan phase for the noncommutative $r_{1} r_{2}$ coordinates as

$$
\Phi_{A}(\theta)=\Phi_{A}\left(1+\frac{\theta \mu \lambda_{e}}{c s^{\prime}}-\frac{\theta d \lambda_{m}}{c s^{\prime \prime}}\right) .
$$

Obviously, the AC and HMW phases in noncommutative coordinates follow for $d=0$ and $\mu=0$, respectively.

\subsection{Particle interacting with a noncommutative gauge field}

In the non-Abelian physical systems considered above the gauge fields $\mathcal{A}$ were internal. However, one can also deal with external gauge fields. When external non-Abelian gauge fields are considered the field strength (11) can be inserted into (37) to obtain the related quantum phase in noncommutative space. Moreover, one can generalize this procedure to the noncommutative $U(N)$-valued gauge fields $\tilde{\mathcal{A}}_{\alpha}=\tilde{\mathcal{A}}_{\alpha}^{n} T_{n}$ by replacing the ordinary product with the star product, so that the field strength is given by

$$
\tilde{F}_{\alpha \beta}=\frac{\partial \tilde{\mathcal{A}}_{\beta}}{\partial r^{\alpha}}-\frac{\partial \tilde{\mathcal{A}}_{\alpha}}{\partial r^{\beta}}-\frac{i \rho}{\hbar}\left(\tilde{\mathcal{A}}_{\alpha} \star \tilde{\mathcal{A}}_{\beta}-\tilde{\mathcal{A}}_{\beta} \star \tilde{\mathcal{A}}_{\alpha}\right) .
$$


The star product is defined in terms of another constant, antisymmetric parameter $\tilde{\theta}_{\alpha \beta}$ as

$$
\star=\exp \left[i \tilde{\theta}_{\alpha \beta}\left(\frac{\overleftarrow{\partial}}{\partial r^{\alpha}} \frac{\vec{\partial}}{\partial r^{\beta}}\right)\right] .
$$

We distinguish the noncommutativity parameter of the space $\theta$ from the noncommutative parameter of the gauge group $\tilde{\theta}$, because in quantum mechanical systems noncommutativity of space does not imply automatically that the related gauge groups should be noncommutative. Obviously, one can let the deformation parameters $\theta$ and $\tilde{\theta}$ to be the same by taking care of the approximations regarding $\theta$-dependent terms.

Let us deal with the noncommutative $U(1)$ where the canonical Hamiltonian is $H_{0}=p^{2} / 2 m$ and the coupling constant is $\rho=e / c$. By inserting (45) into (37) one can obtain the deformed quantum phase for an infinitesimal loop as

$$
\Phi_{N C}(\theta)=\frac{e}{2 \hbar c}\left(\tilde{F}_{\alpha \beta}-\frac{e}{c}(\theta \tilde{F} \tilde{F})_{\alpha \beta}\right) s_{\epsilon}^{\alpha \beta} .
$$

For $\theta=0$ but $\tilde{\theta} \neq 0$ this coincides with the quantum phase obtained in [21] considering a gauge covariant generalization of the $\mathrm{AB}$ phase in noncommutative space.

\section{Discussions}

The most known examples of quantum systems where coordinates are effectively noncommutative are the charged particles moving on a plane in the presence of a strong perpendicular magnetic field and the strings moving in the Neveu-Schwarz background field. In both of these systems noncommutativity parameter is proportional to the inverse of the field involved. Thus for quantum mechanical systems in noncommutative coordinates the value of the noncommutativity parameter $\theta$ depends on the system considered. By adopting this interpretation we would like to derive upper limits on the value of $\theta$ taking into account the quantum phases where experimental verifications are available. However, before proceeding note that semiclassical brackets like Poisson brackets, possess a dimension of $\hbar^{-1}$. Hence, if we demand that the dimension of the noncommutativity parameter $\theta$ be (length) ${ }^{2}$, (14) dictates that we should perform the rescaling

$$
\theta_{\alpha \beta} \rightarrow \frac{\theta_{\alpha \beta}}{\hbar}
$$

In [6] the observed value of the $\mathrm{AB}$ phase reported in [22] was used to derive an upper bound for the noncommutativity parameter $\theta_{A B}$. We adopt the same approach: let $\Delta_{A B}$ denote percentage of the error in measuring the $\mathrm{AB}$ phase. Comparing it with the $\mathrm{AB}$ phase in noncommutative coordinates (39) after the rescaling (48), one obtains the bound

$$
\theta_{A B} \leq \frac{\hbar c}{e B} \Delta_{A B}=\left(\frac{4.1}{2 \pi} \times 10^{-7} \text { Gauss } \mathrm{cm}^{2}\right) \frac{\Delta_{A B}}{B} .
$$

Hence, the noncommutativity parameter regarding the $\mathrm{AB}$ effect $\theta_{A B}$ is proportional to the inverse of the magnetic field $B$. Experimental error reported in 22$]$ is $\Delta_{A B}=20 \%$ and the order of magnitude of the magnetic field is $B \approx 10^{4}$ Gauss. Therefore, we get the bound

$$
\sqrt{\theta_{A B}} \leq 10^{-6} \mathrm{~cm} .
$$

By setting $d=0$ in (44) one obtains the AC phase in noncommutative coordinates after the rescaling (48) as

$$
\Phi_{A C}(\theta)=\Phi_{A C}\left(1+\frac{\theta \mu \lambda_{e}}{\hbar c s^{\prime}}\right)
$$

where $\Phi_{A C}=\frac{\mu \lambda_{e}}{\hbar c}$ is the ordinary AC phase. Now, in terms of the experimental error percentage $\Delta_{A C}$ we get the bound on the noncommutativity parameter related to the AC phase as

$$
\theta_{A C} \leq \frac{s^{\prime}}{\Phi_{A C}} \Delta_{A C}
$$

Considering neutrons, the AC phase was measured in $[23]$ as

$$
\Phi_{A C}^{(n)}=2.19 \pm 0.52 \mathrm{mrad} .
$$


If we let $s^{\prime}$ be of the order of the area enclosed within the path of the neutrons given in $\left[9\right.$ as $s^{\prime} \approx 3 \mathrm{~cm}^{2}$, we get

$$
\sqrt{\theta_{A C}^{(n)}} \leq 17 \mathrm{~cm}
$$

The AC phase was measured in 24] dealing with a superposition of two coherent beams of atoms possessing different magnetic moments as

$$
\Phi_{A C}^{(a)}=2.22 \pm 0.11 \mathrm{mrad} .
$$

Although, the geometry of the experiment is different from the originally proposed one [2], we take $s^{\prime} \approx 0.8 \mathrm{~cm}^{2}$, due to the fact that the beam travels in a region of diameter $\approx 1 \mathrm{~cm}$. Hence, the bound reads

$$
\sqrt{\theta_{A C}^{(a)}} \leq 4 \mathrm{~cm}
$$

The bounds which we obtained (50) and (52) do not coincide with the ones derived in [6] and [9], respectively. And this is because, the quantum phases in noncommutative coordinates which we obtained do not refer to energy of the particles in contrary to the ones attained in the latter approaches.

\section{References}

[1] Y. Aharonov and D. Bohm, Phys. Rev. 115 (1959) 485.

[2] Y. Aharonov and A. Casher, Phys. Rev. Lett. 53 (1984) 319.

[3] X.-G. He and B. H. J. McKellar, Phys. Rev. A 47 (1993) 3424.

M. Wilkens, Phys. Rev. Lett. 72 (1994) 5.

[4] J. Anandan, Phys. Rev. Lett. 85 (2000) 1354.

[5] C. Furtado and C. A. de Lima Ribeiro, Phys. Rev. A 69 (2004) 064104.

[6] M. Chaichian, P. Presnajder, M. M. Sheikh-Jabbari and A. Tureanu, Phys. Lett. B 527 (2002) 149.

[7] J. Gamboa, M. Loewe and J. C. Rojas, "Noncommutativity and the Aharonov-Bohm effect" hep-th/0101081.

[8] Ö. F. Dayi and A. Jellal, J. Math. Phys. 43 (2002) 4592 [Erratum: 45 (2004) 827].

[9] B. Mirza and M. Zarei, Eur. Phys. J. C 32 (2004) 583.

[10] K. Li and F. Wang, Eur. Phys. J. C 50 (2007) 1007.

[11] J. Wang and K. Li, J. Phys. A: Math. Theor. 40 (2007) 2197.

[12] E. Passos, L. R. Ribeiro, C. Furtado and J. R. Nascimento, Phys. Rev. A 76 (2007) 012113.

[13] Ö. F. Dayi, J. Phys. A: Math. Theor. 41 (2008) 315204.

[14] M. V. Berry, Proc. R. Soc. Lond. 392 (1984) 45.

[15] Ö. F. Dayi and M. Elbistan, Phys. Lett. A 373 (2009) 1314, 0806.0707.

[16] G. Spavieri, Phys. Lett. A 310 (2003) 13; Eur. Phys. J D 37 (2006) 327.

[17] T. Guhr and S. Keppeler, Annals of Phys. 322 (2007) 287.

[18] A. Wagh and V. C. Rakhecha, Phys. Rev. Lett 78 (1997) 1399.

[19] S. A. R. Horsley and M. Babiker, J. Phys. B: At. Mol. Opt. Phys. 40 (2007) 2003.

[20] J. Anandan, Phys. Rev. D 15 (1977) 1448; Int. J. Theor. Phys. 19 (1980) 537.

[21] M. Chaichian, M. Langvik, S. Sasaki and A. Tureanu, Phys. Lett. B 666 (2008) 199. 
[22] A. Tonomura, T. Matsuda, R. Suzuki, A. Fukuhara and N. Osakabe, Phys. Rev. Lett. 48 (1982) 1443.

[23] A. Cimmino, G. I. Opat, A. G. Klein, H. Kaise, S. A. Werner, M. Arif and R. Clothier, Phys. Rev. Lett. 63 (1989) 380.

[24] K. Sangster, E. A. Hinds ,S. M. Barnett and E. Riis, Phys. Rev. Lett. 71 (1993) 3641; K. Sangster, E. A. Hinds ,S. M. Barnett, E. Riis and A.G. Sinclair, Phys. Rev. A 51 (1995) 1776. 